\title{
Optimum Times for Step-Stress Cumulative Exposure Model Using Log-Logistic Distribution with Known Scale Parameter
}

\author{
Abedel-Qader Al-Masri and Mohammed Al-Haj Ebrahem \\ Department of Statistics, Yarmouk University, Irbid-Jordan
}

\begin{abstract}
In this paper we assume that the life time of a test unit follows a log-logistic distribution with known scale parameter. Tables of optimum times of changing stress level for simple step-stress plans under a cumulative exposure model are obtained by minimizing the asymptotic variance of the maximum likelihood estimator of the model parameters at the design stress with respect to the change time.
\end{abstract}

Zusammenfassung: In diesem Aufsatz wird angenommen, dass die Lebensdauer einer Testeinheit einer log-logistischen Verteilung mit bekanntem Skalenparameter genügt. Tabellen für die optimalen Zeitpunkte eines Wechsels des Belastungsniveaus für einfache step-stress Pläne unter einem kumulativen Expositionsmodells erhält man durch Minimieren der asymptotischen Varianz des Maximum Likelihood Schätzers der Modellparameter zur zulässigen Spannung bezüglich der Wechselzeit.

Keywords: Maximum Likelihood Estimation, Simple Step-Stress.

\section{Introduction}

The log-logistic distribution arises in a variety of fields. The special features of this distribution together with its relation with the logistic distribution allowed to use it as a model in various real life applications.

Accelerated life tests are used to quickly obtain information on the life time distribution of products by testing them at higher than nominal levels of stress to induce early failures. One way of applying stress to the test units is a step-stress scheme which allows the stress of a unit to be changed at specified times. Nelson (1980) described this important type of accelerated life test. In step-stress testing, a unit is placed on a test at an initial low stress, if it does not fail in a predetermined time $\tau$, stress is increased. If there is a single change of stress, the accelerated life test is called a simple step-stress test.

The cumulative exposure model defined by Nelson (1990) for simple step-stress testing with low stress $X_{1}$ and high stress $X_{2}$ is

$$
G(t)= \begin{cases}G_{1}(t), & t \leq \tau \\ G_{2}(t-\tau+s), & t>\tau\end{cases}
$$

where $G_{i}(t)$ is the cumulative distribution function (cdf) of the failure time at stress $X_{i}, \tau$ is the time to change stress, and $s$ is the solution of $G_{1}(\tau)=G_{2}(s)$.

In the literature, Miller and Nelson (1983) obtained the optimum simple step-stress accelerated life test plans for the case where the test units have exponentially distributed 
life times. Bai, Kim, and Lee (1989) extended the results of Miller and Nelson (1983) to the case of censoring. Khamis and Higgins (1996a) obtained the optimum 3-step stepstress using the exponential distribution. Khamis and Higgins (1996b, 1998) proposed a new model known as KH model for step-stress accelerated life test as an alternative to the Weibull cumulative exposure model. Alhadeed and Yang (2005) obtained the optimum design for the lognormal simple step-stress model. Xiong (1998) presented the inferences of parameters in the simple step-stress model in accelerated life testing with type two censoring. Xiong and Milliken (2002) studied statistical models in step-stress accelerated life testing when stress change times are random and obtained the marginal life time distribution for test units. Nonparametric approaches for step-stress testing have been proposed Schmoyer (1991). Al-Haj Ebrahem and Al-Masri (2007a) obtained optimum design for the log-logistic simple-step stress plan by minimizing the asymptotic variance of the maximum likelihood estimator of a given p-quantile of the life time distribution. Al-Haj Ebrahem and Al-Masri (2007b) obtained the optimum simple-stress plans for Loglogistic distribution when a prespecified censoring time is introduced. For more details see Chung and Bai (1998), Gouno (2001), Xiong and Milliken (2002).

In this paper we obtain tables of optimum times of changing stress levels under a loglogistic cumulative exposure model for a wide range of values of the model parameters. The paper is organized as follows: model and assumptions are described in Section 2, optimum test plan and maximum likelihood estimators of the model parameters are derived in Section 3, conclusions and tables of optimum times of changing stress levels are presented in Section 4.

\section{Model and Assumptions}

The cdf of a log-logistic distribution with parameters $\mu$ and $\sigma$ is

$$
G(t)=\Psi_{\operatorname{logis}}\left(\frac{\log (t)-\mu}{\sigma}\right), \quad 0<t<\infty
$$

where $\Psi_{\text {logis }}(h)=1 /\left(1+e^{-h}\right)$ is the cdf of the logistic distribution with $\mu=0$ and $\sigma=1$. We make the following assumptions:

1. Under any stress the life time of a test unit follows a log-logistic distribution with known scale parameter $\sigma$.

2. Testing is done at two stresses $X_{1}$ and $X_{2}$, with $X_{1}<X_{2}$.

3. The relationship between the mean of the log life time $\mu_{i}$ and the stress $X_{i}$ is $\mu_{i}=$ $\beta_{0}+\beta_{1} X_{i}$, where $\beta_{0}$ and $\beta_{1}$ are unknown parameters to be estimated from the test data. This relationship holds for design stresses between $X_{0}$ and $X_{2}$.

4. The lifetimes of test units are independent and identically distributed.

5. All $n$ units are initially placed on low stress $X_{1}$ and run until time $\tau$ when the stress is changed to high stress $X_{2}$. At $X_{2}$ testing continues until all remaining units fail. 
It is easy to verify that the log-logistic cumulative exposure model for simple step-stress is

$$
G(t)= \begin{cases}\Psi_{\operatorname{logis}}\left(\frac{\log (t)-\mu_{1}}{\sigma}\right) & 0<t \leq \tau . \\ \Psi_{\operatorname{logis}}\left(\frac{\log \left(t-\tau+\tau e^{\mu_{2}-\mu_{1}}\right)-\mu_{2}}{\sigma}\right) & \tau<t<\infty .\end{cases}
$$

Let $Y=\log (T)$ then the cumulative exposure model and the corresponding probability density function (pdf) of $Y$ are

$$
\begin{aligned}
G(y) & =\Psi_{\text {logis }}\left(\phi_{i}\right), \quad y \in \Re_{i} \\
g(y) & =\eta_{i}(y) \psi_{\text {logis }}\left(\phi_{i}\right), \quad y \in \Re_{i},
\end{aligned}
$$

for $i=1,2$, where

$$
\begin{aligned}
\Re_{1} & =(-\infty, \log (\tau)], & \Re_{2}=(\log (\tau), \infty), & \\
\phi_{i} & =\frac{\log \left(\xi_{i}\right)-\mu_{i}}{\sigma}, & \xi_{i} & =e^{y}-(i-1) \delta, \\
\delta & =\tau\left(1-e^{\mu_{2}-\mu_{1}}\right), & \mu_{i} & =\beta_{0}+\beta_{1} X_{i}, \quad \eta_{i}(y)=\frac{e^{y}}{\sigma \xi_{i}} .
\end{aligned}
$$

In expression (4), $\psi_{\text {logis }}(h)=e^{-h} /\left(1+e^{-h}\right)^{2}$ is the pdf of the logistic distribution with $\mu=0$ and $\sigma=1$.

Note that we can use an alternative expression for $\psi_{\operatorname{logis}}(h)$ in terms of hyperbolic secant, since we can write

$$
\psi_{\text {logis }}(h)=\frac{1}{\left(e^{h / 2}\left(1+e^{-h}\right)\right)^{2}}=\frac{1}{\left(e^{h / 2}+e^{-h / 2}\right)^{2}} .
$$

Now, the hyperbolic cosine is defined as $\cosh (h / 2)=\left(e^{h / 2}+e^{-h / 2}\right) / 2$, thus

$$
\psi_{\text {logis }}(h)=\frac{1}{(2 \cosh (h / 2))^{2}} .
$$

Since the hyperbolic secant is defined as $\operatorname{sech}(h / 2)=1 / \cosh (h / 2)$, we write

$$
\psi_{\text {logis }}(h)=\frac{1}{4} \operatorname{sech}^{2}(h / 2) \text {. }
$$

\section{Maximum Likelihood Estimation and Optimum Plans}

Let $y_{i j}, i=1,2, j=1, \ldots, n_{i}$, be the logarithm of the observed life time of test unit $j$ under stress level $i$, where $n_{1}$ denotes the number of units failed at the low stress $X_{1}$ and $n_{2}$ is the number of units failed at the high stress $X_{2}$. The pdf (4) can be written in terms of the hyperbolic secant as

$$
g(y)= \begin{cases}\frac{1}{4 \sigma} \operatorname{sech}^{2}\left(\frac{y-\mu_{1}}{2 \sigma}\right), & -\infty<y \leq \log (\tau) \\ \frac{e^{y}}{4 \sigma\left(e^{y}-\tau+\tau e^{\mu_{2}-\mu_{1}}\right)} \operatorname{sech}^{2}\left(\frac{\log \left(e^{y}-\tau+\tau e^{\mu_{2}-\mu_{1}}\right)-\mu_{2}}{2 \sigma}\right), & \log (\tau)<y<\infty\end{cases}
$$


The likelihood function is

$$
L\left(\beta_{0}, \beta_{1} \mid \underline{y}, \sigma\right)=\prod_{i=1}^{2} \prod_{j=1}^{n_{i}} \eta_{i j}\left(y_{i j}\right) \psi_{\text {logis }}\left(\phi_{i j}\right),
$$

where $\phi_{i j}=\left(\log \left(\xi_{i j}\right)-\mu_{i}\right) / \sigma, \eta_{i j}\left(y_{i j}\right)=e^{y_{i j}} / \sigma \xi_{i j}$, and $\xi_{i j}=e^{y_{i j}}-(i-1) \delta$. The $\log$-likelihood function is given by

$$
\log \left(L\left(\beta_{0}, \beta_{1} \mid \underline{y}, \sigma\right)\right)=C+\sum_{i=1}^{2} \sum_{j=1}^{n_{i}}\left(\pi\left(y_{i j}, \beta_{0}, \beta_{1}\right)-\varpi\left(y_{i j}, \beta_{1}\right)\right),
$$

where $C=\sum_{i=1}^{2} \sum_{j=1}^{n_{i}} y_{i j}-\left(n_{1}+n_{2}\right) \log (4 \sigma), \pi\left(y_{i j}, \beta_{0}, \beta_{1}\right)=2 \log \left(\operatorname{sech}\left(\phi_{i j} / 2\right)\right)$, and $\varpi\left(y_{i j}, \beta_{1}\right)=\log \left(\xi_{i j}\right)$.

The maximum likelihood estimates $\hat{\beta}_{0}$ and $\hat{\beta}_{1}$ for the model parameters $\beta_{0}$ and $\beta_{1}$ are obtained by solving the partial derivatives of the log-likelihood function

$$
\begin{aligned}
\frac{\partial}{\partial \beta_{0}} \log \left(L\left(\beta_{0}, \beta_{1} \mid \underline{y}, \sigma\right)\right) & =\frac{1}{\sigma} \sum_{i=1}^{2} \sum_{j=1}^{n_{i}} \vartheta_{i j}, \\
\frac{\partial}{\partial \beta_{1}} \log \left(L\left(\beta_{0}, \beta_{1} \mid \underline{y}, \sigma\right)\right) & =\frac{1}{\sigma}\left(\sum_{i=1}^{2} \sum_{j=1}^{n_{i}} X_{i} \vartheta_{i j}-\left(X_{2}-X_{1}\right)(\tau-\delta) \sum_{j=1}^{n_{2}} \frac{\vartheta_{2 j}+\sigma}{\xi_{2 j}}\right),
\end{aligned}
$$

where $\vartheta_{i j}=\tanh \left(\phi_{i j} / 2\right)$.

The Fisher information matrix $F$ is obtained by taking the negative expected value of the second partial and mixed partial derivatives of the log-likelihood function with respect to $\beta_{0}$ and $\beta_{1}$. Let

$$
\begin{aligned}
\nabla & =\frac{\tau^{2 / \sigma}}{3} \frac{3 e^{\mu_{1} / \sigma}+\tau^{1 / \sigma}}{\left(e^{\mu_{1} / \sigma}+\tau^{1 / \sigma}\right)^{3}}, \quad \theta=e^{\mu_{2}-\mu_{1}}, \\
\xi & =e^{y}-\tau(1-\theta), \quad \phi=\frac{\log (\xi)-\mu_{2}}{\sigma}, \quad \Delta=e^{y} \operatorname{sech}^{4}(\phi / 2), \\
\omega & =\sigma^{2}(1+\cosh (\phi))+\sigma \sinh (\phi),
\end{aligned}
$$

then the Fisher information matrix is

$$
F=\frac{n}{8 \sigma^{2}}\left[\begin{array}{ll}
F_{11} & F_{12} \\
F_{21} & F_{22}
\end{array}\right]
$$

where

$$
\begin{aligned}
F_{11}= & \frac{1}{3} \\
F_{12}= & X_{1}\left(8 \sigma \nabla+\theta \tau \int_{\Re_{2}} \frac{\Delta}{\xi^{2}} d y\right)+X_{2}\left(\int_{\Re_{2}} \frac{\left(e^{y}-\tau\right) \Delta}{\xi^{2}} d y\right) \\
F_{22}= & X_{1}^{2}\left(8 \sigma \nabla+\theta \tau \int_{\Re_{2}} \frac{\tau \theta+\left(e^{y}-\tau\right) \omega}{\xi^{3}} \Delta d y\right)-X_{1} X_{2} 2 \theta \tau\left(\int_{\Re_{2}} \frac{\left(e^{y}-\tau\right)(\omega-1)}{\xi^{3}} \Delta d y\right) \\
& +X_{2}^{2}\left(\int_{\Re_{2}} \frac{\left(e^{y}-\tau\right)\left(e^{y}-\tau+\tau \theta \omega\right)}{\xi^{3}} \Delta d y\right) .
\end{aligned}
$$


An optimum test plan can be determined by minimizing, with respect to the change time $\tau$, the asymptotic variance of the maximum likelihood estimator of the model parameters at the design stress $X_{0}$. So, we will use numerical search methods to find the value of $\tau^{*}$ that minimizes $\left(\begin{array}{ll}1 & X_{0}\end{array}\right) F^{-1}\left(\begin{array}{ll}1 & X_{0}\end{array}\right)^{t}$. Tables of optimum times $\tau^{*}$ of changing stress levels for different values of $X_{1}, X_{2}, \mu_{1}$, and $\sigma$ are provided in the next section.

\section{Tables of Optimum Times of Changing Stress Levels}

Optimum times $\tau^{*}$ of changing stress levels are presented in Tables 1 to 4 for $X_{1}=$ $0.1,0.3,0.5,0.7,0.9, X_{2}=0.2,0.4,0.6,0.8,1, \mu_{1}=1,2,3$, and $\sigma=1,1.5,2,2.5$. From these tables we can conclude that

1. given $X_{1}, X_{2}$, and $\mu_{1}$, the optimum time $\tau^{*}$ increases as $\sigma$ increases;

2. given $X_{1}, X_{2}$, and $\sigma$, the optimum time $\tau^{*}$ increases as $\mu_{1}$ increases;

3. given $X_{1}, \mu_{1}$, and $\sigma$, the optimum time $\tau^{*}$ increases as $X_{2}$ increases;

4. given $X_{2}, \mu_{1}$, and $\sigma$, the optimum time $\tau^{*}$ decreases as $X_{1}$ increases.

There are still some possibilities of future work. We may use more than two stress levels, for example a three step stress model and obtain the optimum times of changing stress levels. We may allow type I or type II censored data. Also, we may obtain different types of interval estimation, for example large sample confidence intervals, bootstrap confidence intervals and compare them in terms of their lengths and coverage probabilities. It will be also interesting to evaluate the gain in efficiency in testing and precision in parameter estimation in using censoring and additional stresses.

Table 1: Optimum times of changing stress when $\sigma=1.0$.

\begin{tabular}{rrrrrrr}
\hline & \multicolumn{5}{c}{$X_{2}$} \\
\hline$X_{1}$ & $\mu_{1}$ & 0.2 & 0.4 & 0.6 & 0.8 & 1.0 \\
\hline \multirow{3}{*}{0.1} & 1 & 3.3 & 6.0 & 8.2 & 10.0 & 11.6 \\
& 2 & 8.8 & 16.3 & 22.2 & 27.2 & 31.5 \\
& 3 & 24.0 & 44.2 & 60.3 & 73.8 & 85.7 \\
\hline \multirow{3}{*}{0.3} & 1 & & 2.2 & 3.3 & 4.2 & 5.2 \\
& 2 & & 5.9 & 8.8 & 11.5 & 14.0 \\
& 3 & & 16.0 & 24.0 & 31.4 & 38.1 \\
\hline \multirow{3}{*}{0.5} & 1 & & & 1.9 & 2.6 & 3.3 \\
& 2 & & & 5.3 & 7.1 & 8.8 \\
& 3 & & & 14.3 & 19.3 & 24.0 \\
\hline \multirow{3}{*}{0.7} & 1 & & & & 1.8 & 2.3 \\
& 2 & & & & 5.0 & 6.3 \\
& 3 & & & & 13.6 & 17.2 \\
\hline \multirow{3}{*}{0.9} & 1 & & & & & 1.8 \\
& 3 & & & & & 4.9 \\
& 3 & & & & & 13.2 \\
\hline
\end{tabular}


Table 2: Optimum times of changing stress when $\sigma=1.5$.

\begin{tabular}{rrrrrrr}
\hline & & & & $X_{2}$ & \\
\hline$X_{1}$ & $\mu_{1}$ & 0.2 & 0.4 & 0.6 & 0.8 & 1.0 \\
\hline \multirow{3}{*}{0.1} & 1 & 4.6 & 11.5 & 18.1 & 24.4 & 30.3 \\
& 2 & 12.6 & 31.3 & 49.3 & 66.3 & 82.5 \\
& 3 & 34.3 & 85.2 & 134.0 & 180.2 & 224.2 \\
\hline \multirow{3}{*}{0.3} & 1 & & 2.5 & 4.6 & 6.9 & 9.2 \\
& 2 & & 6.7 & 12.6 & 18.9 & 25.1 \\
& 3 & & 18.3 & 34.3 & 51.3 & 68.3 \\
\hline \multirow{3}{*}{0.5} & 1 & & & 2.1 & 3.3 & 4.6 \\
& 2 & & & 5.7 & 9.0 & 12.6 \\
& 3 & & & 15.4 & 24.5 & 34.3 \\
\hline \multirow{3}{*}{0.7} & 1 & & & & 1.9 & 2.8 \\
& 2 & & & & 5.2 & 7.5 \\
& 3 & & & & 14.2 & 20.5 \\
\hline \multirow{3}{*}{0.9} & 1 & & & & & 1.8 \\
& 2 & & & & & 5.0 \\
& 3 & & & & & 13.6 \\
\hline
\end{tabular}

Table 3: Optimum times of changing stress when $\sigma=2.0$.

\begin{tabular}{rrrrrrr}
\hline & & & & $X_{2}$ & & \\
\hline$X_{1}$ & $\mu_{1}$ & 0.2 & 0.4 & 0.6 & 0.8 & 1.0 \\
\hline & 1 & 6.9 & 23.0 & 41.6 & 61.4 & 81.9 \\
0.1 & 2 & 18.7 & 62.4 & 113.2 & 167.0 & 222.6 \\
& 3 & 50.8 & 169.7 & 307.7 & 454.0 & 605.2 \\
\hline \multirow{3}{*}{0.3} & 1 & & 2.9 & 6.9 & 11.8 & 17.2 \\
& 2 & & 8.0 & 18.7 & 31.9 & 46.7 \\
& 3 & & 21.7 & 50.8 & 86.8 & 127.0 \\
\hline \multirow{3}{*}{0.5} & 1 & & & 2.3 & 4.4 & 6.9 \\
& 2 & & & 6.3 & 11.9 & 18.7 \\
& 3 & & & 17.1 & 32.2 & 50.8 \\
\hline \multirow{3}{*}{0.7} & 1 & & & & 2.1 & 3.4 \\
& 2 & & & & 5.6 & 9.3 \\
& 3 & & & & 15.3 & 25.2 \\
\hline \multirow{3}{*}{0.9} & 1 & & & & & 1.9 \\
& 2 & & & & & 5.3 \\
& 3 & & & & & 14.3 \\
\hline
\end{tabular}

\section{Acknowledgements}

The authors would like to thank the editor and the referees for their valuable comments and suggestions that improved the content and style of the paper. 
Table 4: Optimum times of changing stress when $\sigma=2.5$.

\begin{tabular}{rrrrrrr}
\hline & \multicolumn{5}{c}{$X_{2}$} \\
\hline$X_{1}$ & $\mu_{1}$ & 0.2 & 0.4 & 0.6 & 0.8 & 1.0 \\
\hline \multirow{3}{*}{0.1} & 1 & 10.4 & 46.6 & 97.4 & 157.6 & 224.9 \\
& 2 & 28.2 & 126.7 & 264.7 & 428.3 & 611.4 \\
& 3 & 76.6 & 344.5 & 719.4 & 1164.3 & 1662.0 \\
\hline \multirow{3}{*}{0.3} & 1 & & 3.5 & 10.4 & 20.3 & 32.5 \\
& 2 & & 9.6 & 28.2 & 55.1 & 88.4 \\
& 3 & & 26.0 & 76.6 & 149.8 & 240.3 \\
\hline \multirow{4}{*}{0.5} & 1 & & & 2.6 & 5.8 & 10.4 \\
& 2 & & & 7.1 & 15.9 & 28.2 \\
& 3 & & & 19.2 & 43.1 & 76.6 \\
\hline \multirow{3}{*}{0.7} & 1 & & & & 2.2 & 4.3 \\
& 2 & & & & 6.1 & 11.6 \\
& 3 & & & & 16.6 & 31.6 \\
\hline \multirow{3}{*}{0.9} & 1 & & & & & 2.1 \\
& 3 & & & & & 5.6 \\
& & & & & & \\
\hline
\end{tabular}

\section{References}

Al-Haj Ebrahem, M., and Al-Masri, A. (2007a). Optimum simple step-stress plan for loglogistic cumulative exposure model. Metron - International Journal of Statistics, $L X V, 23-34$.

Al-Haj Ebrahem, M., and Al-Masri, A. (2007b). Optimum simple step-stress plans for log-logistic distribution under time censoring. Abhath AlYarmouk: Basic Sciences and Engeneering, 16, 319-327.

Alhadeed, A., and Yang, S. (2005). Optimal simple step-stress plan for cumulative exposure model using log-normal distribution. IEEE Transactions on Reliability, 54, 64-68.

Bai, D., Kim, M., and Lee, S. (1989). Optimum simple step-stress accelerated life tests with censoring. IEEE Transactions on Reliability, 38, 528-532.

Chung, S., and Bai, D. (1998). Optimal designs of simple step-stress accelerated life tests for lognormal lifetime distributions. International Journal of Reliability, Quality and Safety Engineering, 5, 315-336.

Gouno, E. (2001). An inference method for temperature step-stress accelerated life testing. Quality and Reliability Engineering International, 17, 11-18.

Khamis, I., and Higgins, J. (1996a). Optimum 3-step step-stress tests. IEEE Transactions on Reliability, 45, 341-345.

Khamis, I., and Higgins, J. (1996b). An alternative to the weibull step-stress model. In Proceedings of the Joint Statistics Meeting, Chicago, USA (p. 123-127). American Statistical Association.

Khamis, I., and Higgins, J. (1998). A new model for step-stress testing. IEEE Transactions on Reliability, 47, 131-134. 
Miller, R., and Nelson, B. (1983). Optimum simple step-stress plans for accelerated life testing. IEEE Transactions on Reliability, 32, 59-65.

Nelson, W. (1980). Accelerated life testing step-stress models and data analysis. IEEE Transactions on Reliability, 29, 103-108.

Nelson, W. (1990). Accelerated Testing, Statistical Models, Test Plans, and Data Analysis. New York: Wiley.

Schmoyer, R. (1991). Nonparametric analysis for two-level single-stress accelerated life tests. Technometrics, 33, 175-186.

Xiong, C. (1998). Inferences on a simple step-stress model with type-II censored exponential data. IEEE Transactions on Reliability, 47, 142-146.

Xiong, C., and Milliken, G. (2002). Prediction for exponential lifetimes based on stepstress testing. Communications in Statistics - Simulation and Computation, 31, 539-556.

Authors' Address

Abedel-Qader Al-Masri and Mohammed Al-Haj Ebrahem

Department of Statistics

Yarmouk University

Irbid-Jordan

E-Mail: almasri68@yu .edu.jo and m_hassanb@hotmail.com 